PREPARED FOR THE U.S. DEPARTMENT OF ENERGY, UNDER CONTRACT DE-AC02-76CH03073

PPPL-3661

PPPL-3661

UC-70

Microinstability Studies for the Large Helical Device

by

G. Rewoldt, L.-P. Ku, W.M. Tang, H. Sugama, N. Nakajima,

K.Y. Watanabe, S. Murakami, H. Yamada, and W.A. Cooper

January 2002

NM|

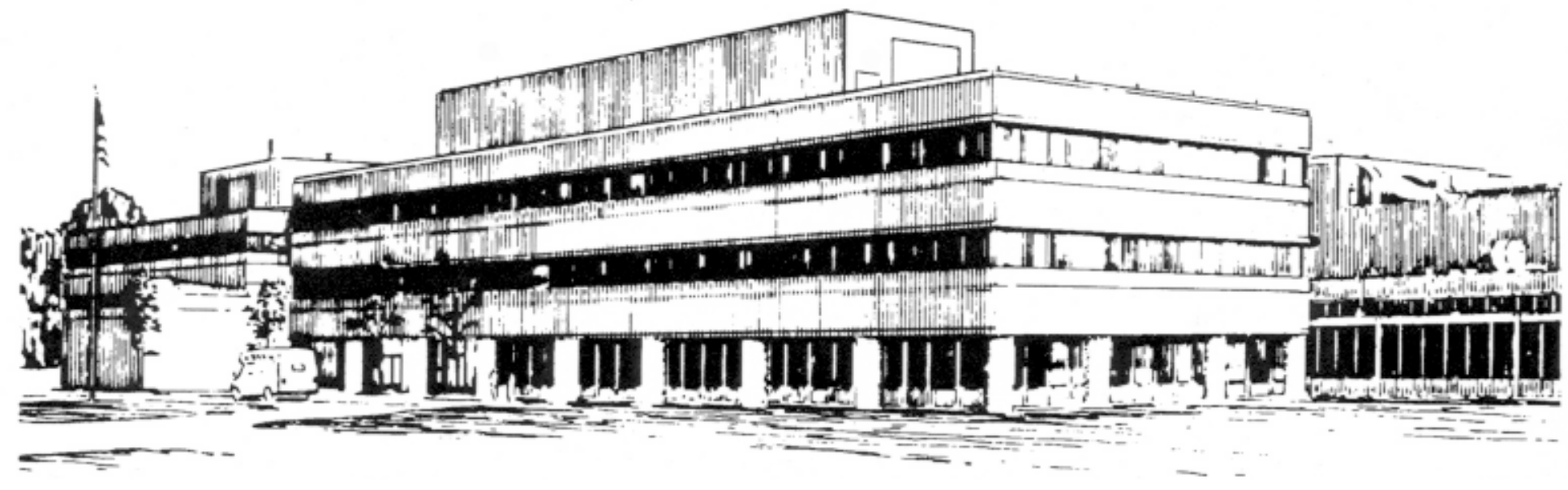

PRINCETON PLASMA PHYSICS LABORATORY PRINCETON UNIVERSITY, PRINCETON, NEW JERSEY 


\section{PPPL Reports Disclaimer}

This report was prepared as an account of work sponsored by an agency of the United States Government. Neither the United States Government nor any agency thereof, nor any of their employees, makes any warranty, express or implied, or assumes any legal liability or responsibility for the accuracy, completeness, or usefulness of any information, apparatus, product, or process disclosed, or represents that its use would not infringe privately owned rights. Reference herein to any specific commercial product, process, or service by trade name, trademark, manufacturer, or otherwise, does not necessarily constitute or imply its endorsement, recommendation, or favoring by the United States Government or any agency thereof. The views and opinions of authors expressed herein do not necessarily state or reflect those of the United States Government or any agency thereof.

\section{Availability}

This report is posted on the U.S. Department of Energy's Princeton Plasma Physics Laboratory Publications and Reports web site in Fiscal Year 2002. The home page for PPPL Reports and Publications is: http://www.pppl.gov/pub_report/

DOE and DOE Contractors can obtain copies of this report from:

U.S. Department of Energy

Office of Scientific and Technical Information

DOE Technical Information Services (DTIS)

P.O. Box 62

Oak Ridge, TN 37831

Telephone: (865) 576-8401

Fax: (865) 576-5728

Email: reports@adonis.osti.gov

This report is available to the general public from:

National Technical Information Service

U.S. Department of Commerce

5285 Port Royal Road

Springfield, VA 22161

Telephone: 1-800-553-6847 or

(703) 605-6000

Fax: (703) 321-8547

Internet: http://www.ntis.gov/ordering.htm 


\title{
Microinstability Studies for the Large Helical Device
}

G. Rewoldt, L.-P. Ku, W. M. Tang

Princeton Plasma Physics Laboratory, Princeton University,

Princeton, New Jersey, United States of America

H. Sugama, N. Nakajima, K. Y. Watanabe, S. Murakami, H. Yamada

National Institute for Fusion Science, Toki, Japan

W. A. Cooper

Centre de Recherche en Physique des Plasmas, Ecole

Polytechnique Fédérale de Lausanne, Lausanne, Switzerland

\begin{abstract}
Fully kinetic assessments of the stability properties of toroidal drift modes have been obtained for cases for the Large Helical Device (LHD). This calculation employs the comprehensive linear microinstability code FULL, as recently extended for nonaxisymmetric systems. The code retains the important effects in the linearized gyrokinetic equation, using the lowest-order "ballooning representation" for high toroidal mode number instabilities in the electrostatic limit. These effects include trapped particles, FLR, transit and bounce and magnetic drift frequency resonances, etc., for any number of plasma species. Results for toroidal drift waves destabilized by trapped electrons and ion temperature gradients are presented, using numerically-calculated three-dimensional MHD equilibria. These are reconstructed from experimental measurements. Quasilinear fluxes of particles and energy for each species are also calculated. Pairs of LHD discharges with different magnetic axis positions and with and without pellet injection are compared.
\end{abstract}




\section{G. Rewoldt, et al.}

\section{Introduction}

A comprehensive kinetic code for linear microinstabilities in tokamaks, the FULL code[1, 2], has been generalized[3] to nonaxisymmetric geometry for application to stellarators. This extended version has previously been applied to the Large Helical Device[4] (LHD) for a single case[5]. Here we apply it to two pairs of LHD discharges, with different magnetic axis positions and with and without pellet injection. This version of the FULL code is interfaced with the three-dimensional MHD equilibrium code VMEC [6, 7], which works in VMEC coordinates. The resulting MHD equilibrium is transformed into Boozer coordinates[8] by the TERPSICHORE code[9], and the quantities needed along a single chosen magnetic field line are constructed by the VVBAL code[10]. The FULL code implements a high- $n$ (toroidal mode number) kinetic calculation employing the lowest-order ballooning representation. It includes trapped particles, finite Larmor radius effects to all orders, banana orbital dynamics, bounce and transit and magnetic drift frequency resonances, equilibrium shaping effects, etc., for all species. The FULL code numerically locates all of the particle turning points along the magnetic field line, so that all classes of trapped particles are included automatically, including both toroidally-trapped and helically-trapped classes. The current nonaxisymmetric version of the FULL code finds linear growth rates and real frequencies in the electrostatic, collisionless limit for any unstable root (not necessarily the most unstable), and also calculates the quasilinear fluxes of particles and energy for each included plasma species, in terms of an externally-specified nonlinear saturation level.

Here, we will compare two pairs of LHD experimental discharges. One pair is matched except for magnetic axis position. The other pair consists of a pellet-injection discharge with a matching no-pellet discharge. These kinds of discharges in LHD are compared from an experimental point of view in Ref. [11]. For all of these discharges, we employ experimentally reconstructed MHD equilibria for LHD, with experimentally derived density and temperature profiles. All of the calculations here include electrons, background hydrogen ions, carbon and oxygen impurity ions, and hot hydrogen beam ions with a slowing-down equilibrium distribution function. All of the other species have a Maxwellian equilibrium distribution function. However, separate ion temperature profiles are not available for these discharges, so we take $T_{j}=T_{e}$ for all species $j$ except the hot beam species. All of the eigenfunctions are mainly localized within the toroidal magnetic field well around $\theta=0$, and overlap only a few helical wells. We choose ballooning parameter $\theta_{0}\left(\equiv \theta_{k}\right)=0$ for all of the cases here. This choice maximizes the linear growth rate. Also, the dependence on the magnetic field line label $\alpha \equiv \zeta-q \theta$ is generally weak for these cases and we arbitrarily choose $\alpha=0$. The linear instabilities considered are the toroidal drift modes (trapped-electron modes (TEM) and ion temperature gradient (ITG) modes). 


\section{Magnetic Axis Shift Comparison}

The first pair of discharges consists of discharge 11369 at $t=2.2 \mathrm{~s}$ and discharge 16727 at $t=$ 1.1 s. Discharge 11369 is inward-shifted with $R_{m a} \simeq 3.60 \mathrm{~m}$, while discharge 16727 is standard with $R_{m a} \simeq 3.75 \mathrm{~m}$. Shot 11369 has better total transport than shot 16727 . The neoclassical transport can account for most of the difference in the core region $\left(s \simeq(r / a)^{2}<0.65\right)$. In other words, the anomalous transport in the core may be comparable in the two shots. The better transport in shot 11369 in the peripheral region $(s>0.65)$ cannot be explained directly by the neoclassical transport differences. The major difference in profiles is that $\eta_{i}^{e} \equiv d \ln T_{i} / d \ln n_{e}=$ $\eta_{e} \equiv d \ln T_{e} / d \ln n_{e}$ is high in the edge region for shot $11369(=2.6$ at $s=0.79)$, while $\eta_{i}^{e}=\eta_{e}$ is smaller for shot $16727,(=1.0$ at $s=0.79)$.

The ballooning representation input function $|B(\theta)|$ on the chosen magnetic field line on a chosen magnetic surface $(s=0.79)$ is shown in Fig. 1(a) for shot 11369, with the ballooning representation [10] form of $k_{\perp}^{2}(\theta)$ shown in Fig. $1(\mathrm{~b})$ and the curvature $k_{\perp} \cdot\{\mathbf{b} \times[(\mathbf{b} \cdot \nabla) \mathbf{b}]\}$ shown in Fig. 1(c). The corresponding functions for shot 16727 are shown in Figs. 1(d)-1(f). There are no drastic differences between the two shots for these input functions, except that the magnetic field strength minima for successive helical wells all have about the same value in Fig. 1(a) for shot 11369. This property is known to generally improve the neoclassical transport[12]. The magnetic field strength in Fig. 1(d) for shot 16727 does not have this property. This can account for some of the differences in neoclassical transport between these two shots. Illustrative orbit extents in $\theta$ are shown in Figs. 1(a) and 1(d) for toroidally and helically trapped particles.

Results are shown for the linear growth rate $\gamma$ and the real frequency $\omega_{r}$ versus $\eta_{i}^{e}=\eta_{e}$ for collisionless electrostatic toroidal drift modes in Fig. 2(a) for $s=0.61$ and $k_{\perp}(\theta=0) \rho_{i}=0.50$ for shot 11369, and in Fig. 2(b) for $s=0.625$ and $k_{\perp}(\theta=0) \rho_{i}=0.24$ for shot 16727. In these figures, the total pressure gradient is held fixed as $\eta_{i}^{e}=\eta_{e}$ varies, so that the density gradient decreases as the electron and ion temperature gradients increase, and vice-versa. Different roots are found for the two shots, namely a TEM-ITG hybrid mode (this hybridization is typical in tokamaks) for shot 11369 , and to separate TEM and ITG modes for shot 16727 . Whether or not hybridization of the TEM and ITG roots will occur generally seems to depend more on the density and temperature profiles, and less on the magnetic structure, for each case. For shot 11369 in Fig. 2(a), as $\eta_{i}^{e}=\eta_{e}$ increases from zero, the growth rate for the TEM-ITG root decreases and then saturates, and the corresponding real frequency moves from the electron to the ion diamagnetic direction. This root is unstable at the experimental value of $\eta_{i}^{e}=\eta_{e}$. For shot 16727 in Fig. 2(b), the growth rate and the real frequency for the TEM root both decrease with increasing $\eta_{i}^{e}=\eta_{e}$, until the mode goes stable. The ITG root is unstable only above a criticial value of $\eta_{i}^{e}=\eta_{e}$, and is the only one unstable at the experimental value of $\eta_{i}^{e}=\eta_{e}$. 


\section{G. Rewoldt, et al.}

The variations of $\gamma$ and $\omega_{r}$ with $k_{\perp}(\theta=0) \rho_{i} \propto n$ are shown in Figs. 2(c) and 2(d) for the TEM-ITG root for shot 11369, and for the ITG mode for shot 16727, with the experimental values of $\eta_{i}^{e}$ and $\eta_{e}$. The eigenfrequency has a growth rate peak for $k_{\perp} \rho_{i}(\theta=0) \simeq 0.2$ for the TEM-ITG and ITG roots for the two shots.

The behavior of $\gamma$ and $\omega_{r}$ with $s$ for both shots is shown in Figs. 2(e) and 2(f). The modes are radially localized to the outer part of the cross-section for both shots, where the density gradient has its normal sign, but are stable in the inner region, where the density gradient is reversed. The real frequencies of the TEM-ITG root for shot 11369 do not vary strongly with radius, while that for the ITG root in shot 16727 makes a transition from the ion to the electron diamagnetic direction with increasing $s$, suggesting that it acquires an increasing trapped-electron contribution with increasing $s$. For $s<0.8$, the growth rate for the TEM-ITG root for shot 11369 is the largest, and for $s>0.8$, the growth rate for the ITG root for shot 16727 is the largest. More of the differences in the results for the two shots appear to come from the differences in density and temperature profile shapes, and thus in the $\eta_{i}^{e}=\eta_{e}$ profiles, than from the differences in magnetic curvature due to the different magnetic axis positions. These growth rate differences possibly suggest a higher level of anomalous transport for shot 16727 in the outermost region $(s>0.8)$ relative to shot 11369 due to these density and temperature profile differences. Effects of multiple-helicity magnetic fields produced by the inward axis shift on the ITG mode, compared with the neoclassical ripple transport, are investigated in Ref. [13], where it is also concluded that the confinement improvement with the inward axis shift cannot be accounted for by results of a linear ITG mode analysis, just from the magnetic field differences.

The FULL code also calculates quasilinear particle fluxes $\Gamma_{j}$ and total energy fluxes $Q_{j}$ for each species. The energy fluxes $Q_{j}$ include the convective part. The electron energy fluxes $Q_{e}$ are always positive (outward). For ratios of fluxes, the unknown nonlinear saturation level for the mode divides out. Flux ratios, normalized to $Q_{e}$, are shown in Fig. 3(a) for shot 11369 and Fig. 3(b) for shot 16727. The electron heat flux (unity on this plot) is dominant at low $\eta_{i}^{e}=\eta_{e}$, and the main ion (hydrogen) heat flux at high $\eta_{i}^{e}=\eta_{e}$. There are reversed (inward) particle fluxes for shot 11369 at high $\eta_{i}^{e}=\eta_{e}$, but not for shot 16727 . An outward energy flux with an inward particle flux for a species means that hot and cold particles of that species are interchanging. The impurity fluxes seem to be small and the hot beam species fluxes seem to be negligable for these cases.

The hollow density profile for shot 11369 reduces $p^{\prime}$ in the interior, below its critical value, because the density gradient and the temperature gradient offset one another. Artificial variation of the local pressure gradient (with a fixed MHD equilibrium) is employed in Fig. 3(c) for $s=0.34$, showing the approach to the marginal value, which is well above the experimental 
value. Artificial variation of the impurity fraction (or $Z_{e f f}$ ) is shown in Fig. 3(d) for shot 11369 , and artificial variation of the impurity density gradient in Fig. 3(e). The expected moderate variation of the growth rates is seen in both of these plots.

In the experimental comparison of these kinds of discharges in Ref. [11], as the magnetic axis radius $R_{m a}$ is decreased from $3.75 \mathrm{~m}$ to $3.60 \mathrm{~m}$, it is seen ( $c f$. Fig. 4(c) there) that there is a larger factor of improvement in the electron thermal conductivity at larger minor radii and a smaller factor of improvement at smaller minor radii (though there is some improvement everywhere). This experimental trend shows some correspondence to the results here, where the case with $R_{m a} \simeq 3.75 \mathrm{~m}$ appears to be the most unstable in the outermost part of the plasma, while the case with $R_{m a} \simeq 3.60$ m appears to be the most unstable somewhat further into the plasma, as seen in Fig. 2(e).

\section{Pellet Injection Comparison}

For the other pair of cases, we use experimentally reconstructed MHD equilibria for LHD shot 11849 at $t=2.565 \mathrm{~s}$ and shot 17120 at $t=4.000 \mathrm{~s}$. Shot 11849 is a pellet shot, and shot 17120 is a non-pellet shot for comparison, both with $R_{m a}=3.6 \mathrm{~m}$. The major difference in profiles is that the hollow region in the electron density, which reduces the total pressure gradient, is much wider for the non-pellet shot, so that the pressure gradient stays large much further in from the edge for the pellet shot than for the non-pellet shot. The ballooning representation input function $|B(\theta)|$ on the chosen field line on the chosen magnetic surface $(s=0.51)$ is shown in Fig. 4(a) for shot 11849, with $k_{\perp}^{2}(\theta)$ in Fig. 4(b) and the curvature $k_{\perp} \cdot\{\mathbf{b} \times[(\mathbf{b} \cdot \nabla) \mathbf{b}]\}$ in Fig. 4(c). The corresponding functions are shown in Figs. 4(d)-4(f) for shot 17120. Illustrative trapped particle regions are shown in Figs. 4(a) and 4(d).

Results are shown for the linear growth rate $\gamma$ and the real frequency $\omega_{r}$ versus $\eta_{i}^{e}=\eta_{e}$ in Figs. $5(\mathrm{a})$ and $5(\mathrm{~b})$, for $s=0.51$ for both shots. One root is found for pellet shot 11849 , corresponding to a TEM-ITG hybrid mode, and two roots for non-pellet shot 17120, corresponding to separate TEM and ITG modes. The growth rates for the TEM-ITG root for shot 11849 and for the TEM root for shot 17120 decrease substantially with increasing $\eta_{i}^{e}=\eta_{e}$ and then saturate. The growth rate for the ITG root for shot 17120 increases gradually above a critical value. At the experimental value of $\eta_{i}^{e}=\eta_{e}$ for shot 17120 , the growth rates of the TEM and ITG roots are comparable. The real frequencies of the TEM root and of the ITG root for shot 17120 remain in the electron and ion diamagnetic directions, respectively, while the real frequency for the TEM-ITG root for shot 11849 makes a transition from the electron to the ion diamagnetic direction with increasing $\eta_{i}^{e}=\eta_{e}$, as is typical for these hybrid roots.

The variation of $\gamma$ and $\omega_{r}$ with $k_{\perp}(\theta=0) \rho_{i}$ or $n$ is shown in Figs. $5(\mathrm{c})$ and $5(\mathrm{~d})$ for the TEM-ITG root for shot pellet 11849 and for the ITG and TEM roots for non-pellet shot 17120 , 


\section{G. Rewoldt, et al.}

for the experimental values of $\eta_{i}^{e}$ and $\eta_{e}$. All three roots have growth rate maxima in the range $k_{\perp}(\theta=0) \rho_{i}=0.2$ to 0.4 . Tokamak cases generally have growth rate maxima for drift modes in the range for $k_{\perp}(\theta=0) \rho_{i}$ from 0.2 to 1.0, and these particular LHD maxima are at the lower end of that typical range, as was also the case for the results in the previous section. The behavior of $\gamma$ and $\omega_{r}$ with $r / a($ i.e., $\sqrt{s})$ for both shots is shown in Figs. $5(\mathrm{e})$ and $5(\mathrm{f})$. The modes are radially localized to the outer part of the cross-section for both shots, where the density gradient has its normal sign, but are stable in the inner region, where the density gradient is reversed. However, the mode extends in further radially for pellet shot 11849, and in general has a higher growth rate in the interior. The flux ratios are shown in Fig. 6(a) for pellet shot 11849 and Fig. 6(b) for non-pellet shot 17120. Again, the electron heat flux is dominant at low $\eta_{i}^{e}=\eta_{e}$, and the main hydrogen ion heat flux at high $\eta_{i}^{e}=\eta_{e}$. There are reversed particle fluxes for both shots at high $\eta_{i}^{e}=\eta_{e}$. Again, the impurity fluxes seem to be small and the hot beam species fluxes seem to be negligable for these cases.

Experimentally, in Ref. [11] (cf. Fig. 6(b) there), it is found that there is a substantially higher level of anomalous electron thermal conductivity deep in the plasma core for a pellet discharge compared to a corresponding gas puff discharge. This corresponds to the result shown here in Fig. 5(e) where the pellet discharge 11849 has a clearly larger linear growth rate level than the corresponding non-pellet shot 17120 in the plasma interior.

\section{Conclusions}

We have converted the existing tokamak (axisymmetric) linear microinstability code FULL to a stellarator (nonaxisymmetric) code, with the same kinetic effects included, in the electrostatic limit. Results have been obtained for linear growth rates and real frequencies, as well as for quasilinear particle and energy fluxes, for collisionless electrostatic toroidal drift modes for four LHD discharges. The most general conclusion here is that there are indeed modes that we calculate to be linearly unstable for these discharges, which could be responsible for the experimentally-observed anomalous transport described in Ref. [11]. The anomalous transport associated with these linear instabilities would have gyro-Bohm scaling, and that is what is mainly observed in the experimental results in Ref. [11] (cf. Fig. 2(d) there), so that these are at least consistent. However, the neoclassical transport also shown in Fig. 2(d) of Ref. [11] has close to gyro-Bohm-like scaling also, except at the very edge of the plasma, where the neoclassical transport has closer to Bohm scaling. In this region, the experimental transport still has gyroBohm scaling, which is thus inconsistent with the neoclassical transport, but is consistent with the anomalous transport that would be expected from the kind of linear instabilities calculated here, and which are generally unstable in this radial region. In particular, unstable TEM and 
ITG roots, as well as TEM-ITG hybrid roots, have been obtained here, which also have some properties in common with tokamak results. Future extensions to the FULL code may include a fully electromagnetic version, inclusion of a rotation model, etc. We would also like to improve the collision model used in the FULL code[3] and further investigate collisional effects, which are normally expected to be stabilizing, on these instabilities in the LHD device. We plan to apply the nonaxisymmetric FULL code to additional cases for LHD and for other existing or planned stellarators.

\section{Acknowledgements}

The authors would like to thank the LHD Experimental Group for fruitful communications. This work was supported by United States Department of Energy Contract No. DE-AC02-76CHO-3073, and in part by a Grant-in-Aid from the Japanese Ministry of Education, Science, and Culture.

\section{References}

[1] Rewoldt, G., Tang, W.M., Chance, M.S., Phys. Fluids 25 (1982) 480.

[2] Rewoldt, G., Tang, W.M., Hastie, R.J., Phys. Fluids 30 (1987) 807.

[3] Rewoldt, G., Ku, L.-P., Tang, W.M., Cooper, W.A., Phys. Plasmas 6 (1999) 4705.

[4] Iiyoshi, A., Komori, A., Ejiri, A., et al., in Proceedings of the 17th International Conference on Plasma Physics and Controlled Nuclear Fusion Research, (Yokohama, Japan, October 1998), Nucl. Fusion 39 (1999) 1245.

[5] Rewoldt, G., Ku, L.-P., Tang, W.M., Sugama, H., Nakajima, N., Watanabe, K.Y., Murakami, S., Yamada, H., Cooper, W.A., Phys. Plasmas 7 (2000) 4942.

[6] Hirshman, S.P., Lee, D.K., Comput. Phys. Commun. 39 (1986) 161.

[7] Hirshman, S.P., Schwenn, U., Nuehrenberg, J., J. Comput. Phys. 87 (1990) 396.

[8] Boozer, A.H., Phys. Fluids 24 (1980) 904.

[9] Anderson, D.V., Cooper, W.A., Gruber, R., Merazzi, S., Schwenn, U., Int. J. Supercomp. Appl. 4 (1990) 34.

[10] Cooper, W.A., Plasma Phys. and Controlled Fusion 34 (1992) 1011.

[11] Yamada, H., Watanabe, K.Y., Yamazaki, K., et al., in Proceedings of the 18th International Conference on Plasma Physics and Controlled Nuclear Fusion Research, (Sorrento, Italy, October 2000), Nucl. Fusion 41 (2001) 901.

[12] Mynick, H.E., Chu, T.K., Boozer, A.H., Phys. Rev. Lett. 48 (1982) 322.

[13] Kuroda, T., Sugama, H., J. Phys. Soc. Jpn. 70 (2001) 2235. 

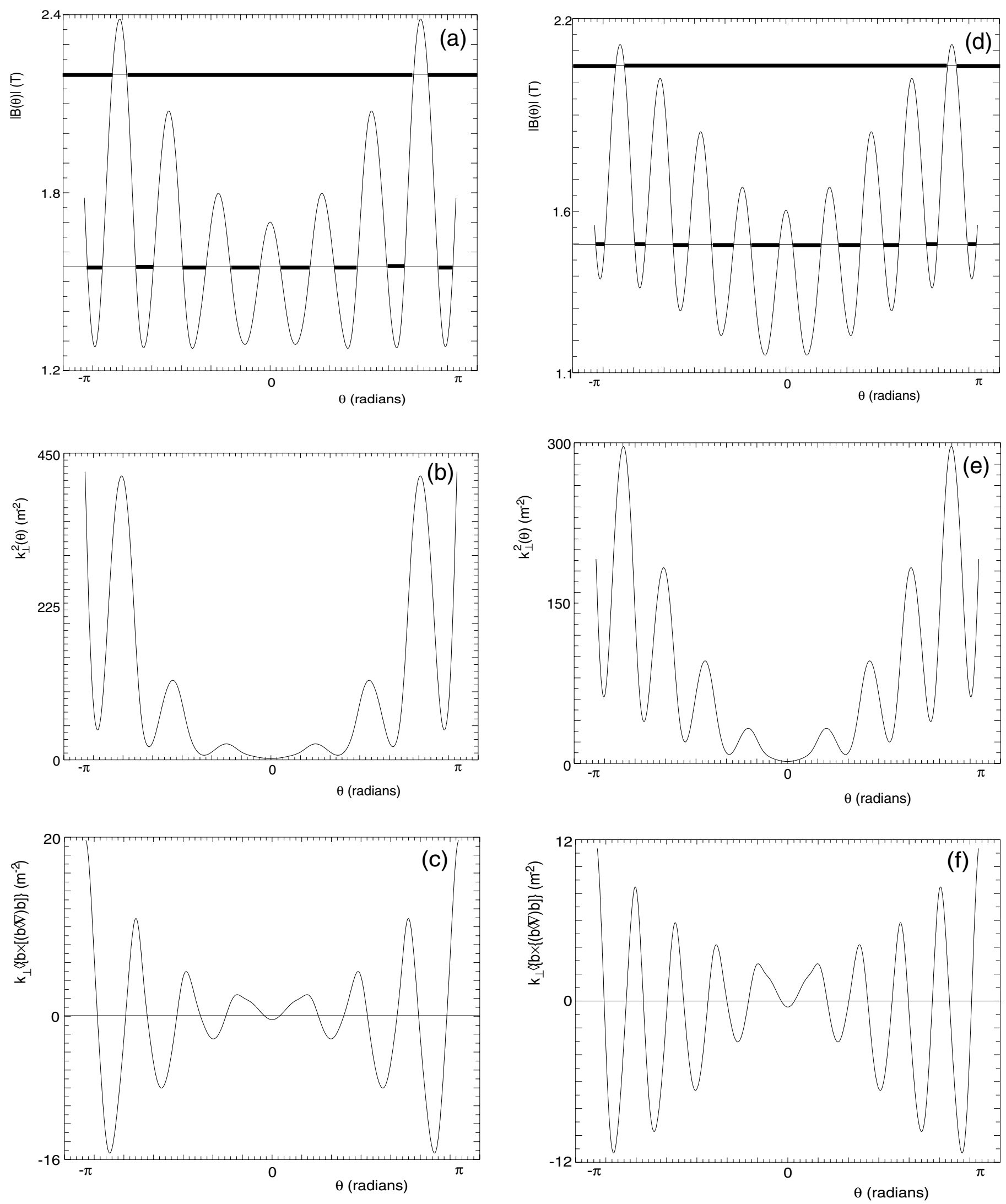

Figure 1. (a) $|B(\theta)|,(b) k_{\perp}^{2}(\theta)$, and (c) curvature for LHD shot 11369 at $t=$ $2.2 s, \alpha=\theta_{0}=0, s \approx(r / a)^{2}=0.79$. (d) $|B(\theta)|,(e) k_{\perp}^{2}(\theta)$, and $(f)$ curvature for shot 16727 at $t=1.1 \mathrm{~s}, \alpha=\theta_{0}=0, s=0.79$. 

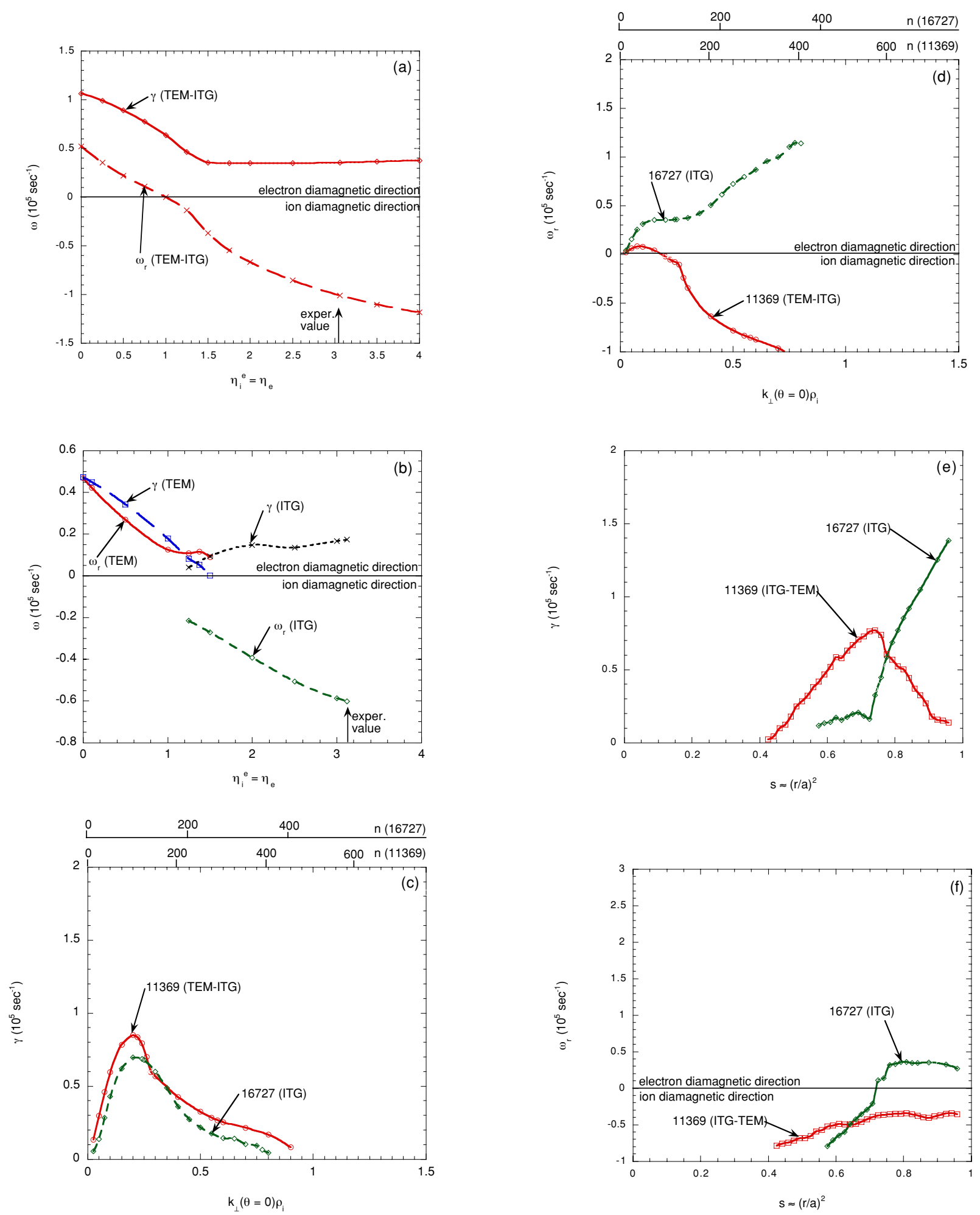

Figure 2. $\omega$ versus $\eta_{i}^{e}=\eta_{e}$ for (a) shot 11369, $s=0.61, k_{\perp}(\theta=0) \rho_{i}=0.50$, (b) shot 16727, $s=0.625, k_{\perp}(\theta=0) \rho_{i}=0.24 ;(c) \gamma$ and $(d) \omega_{r}$ versus $k_{\perp}(\theta=0) \rho_{i} \propto n$ for shots 11369 and 16727 at $s=0.79$; (e) $\gamma$ and $(f) \omega_{r}$ versus s for shots 11369 and 16727 at $k_{\perp}(\theta=0) \rho_{i}=0.24$ and 0.30 . 

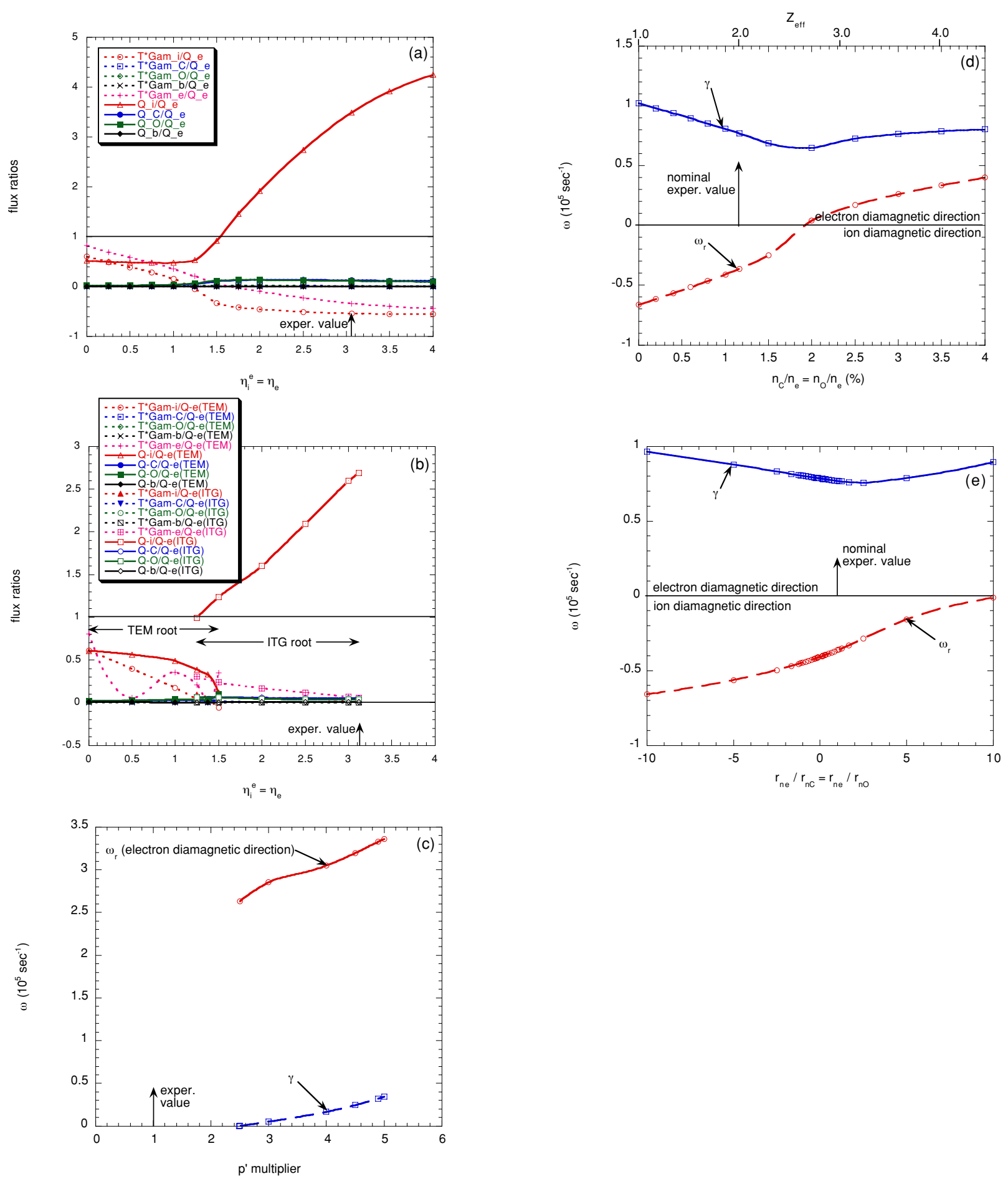

Figure 3. Flux ratios versus $\eta_{i}^{e}=\eta_{e}$ for (a) shot 11369, $k_{\perp}(\theta=0) \rho_{i}$

$=0.50, s=0.61$ for TEM-ITG root, $(b)$ shot 16727 , $k_{\perp}(\theta=0) \rho_{i}=0.24, s=0.625$, for ITG root

(c) flux ratios versus artificial pressure gradient multiplier for shot 11369, $\eta_{i}^{e}=\eta_{e}=-2.2, k_{\perp}(\theta=0) \rho_{i}=0.78, s=0.34$,

(d) $\omega$ versus impurity density fractions for shot 11369, $r_{n C} / r_{n e}=r_{n O} / r_{n e}=$ 1.0, $k_{\perp}(\theta=0) \rho_{i}=0.30, s=0.74$, (e) $\omega$ versus impurity density gradient scale length for shot 11369, $n_{C} / n_{e}=n_{O} / n_{e}=1.163 \%\left(Z_{e f f}=2.0\right)$, $k_{\perp}(\theta=0) \rho_{i}=0.30, s=0.74$ 

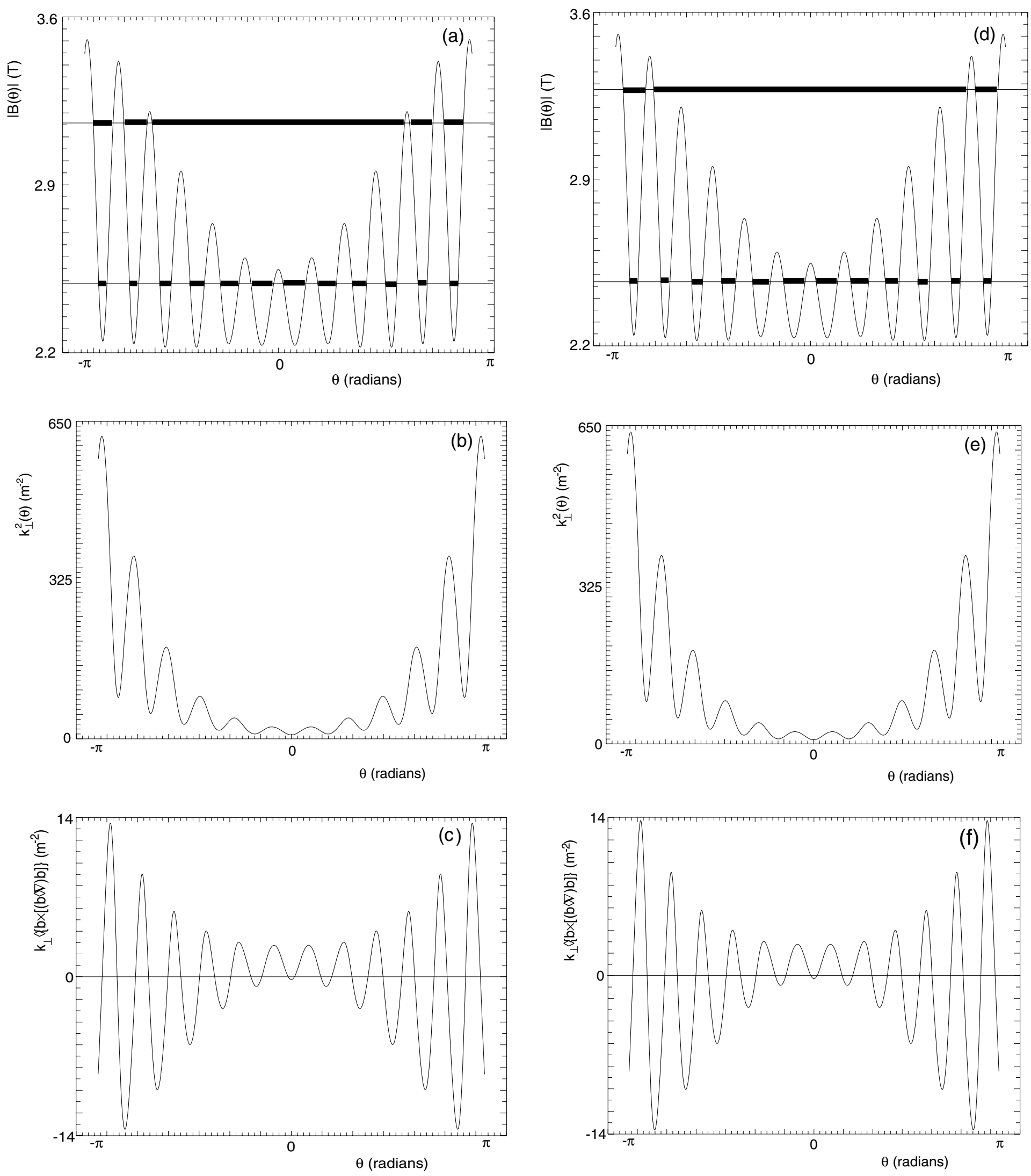

Figure 4. (a) $|B(\theta)|,(b) k_{\perp}^{2}(\theta)$, and (c) curvature for LHD shot 11849 at $t=$ $2.565 s, \alpha=\theta_{0}=0, s \approx(r / a)^{2}=0.51$. (d) $|B(\theta)|$, (e) $k_{\perp}^{2}(\theta)$, and $(f)$ curvature for shot 17120 at $t=4.0 \mathrm{~s}, \alpha=\theta_{0}=0, s=0.51$. 

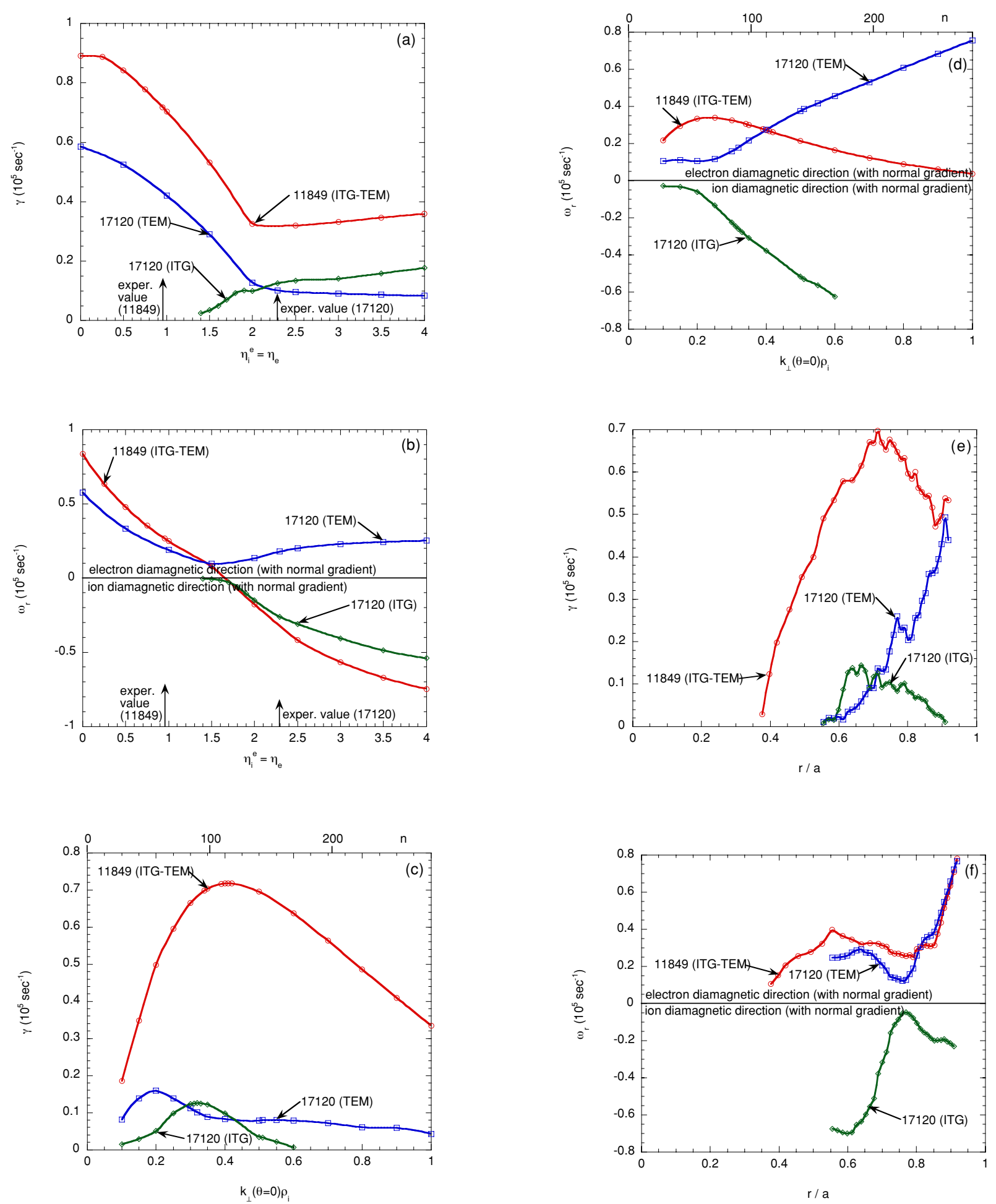

Figure 5. (a) $\gamma$ and (b) $\omega_{r}$ versus $\eta_{i}^{e}=\eta_{e}$ for shots 11849 and 17120, with $k_{\perp}(\theta=0) \rho_{i}=0.41$ and 0.32 , respectively, and $s=0.51$. (c) $\gamma$ and $(d) \omega_{r}$ versus $k_{\perp}(\theta=0) \rho_{i} \propto n$ for shots 11849 and 17120 with $s=0.51$.

(e) $\gamma$ and $(f) \omega_{r}$ versus $r / a=s^{1 / 2}$ for shots 11849 and 17120 with $k_{\perp}(\theta=0) \rho_{i}=0.34$ and 0.32 , respectively. 

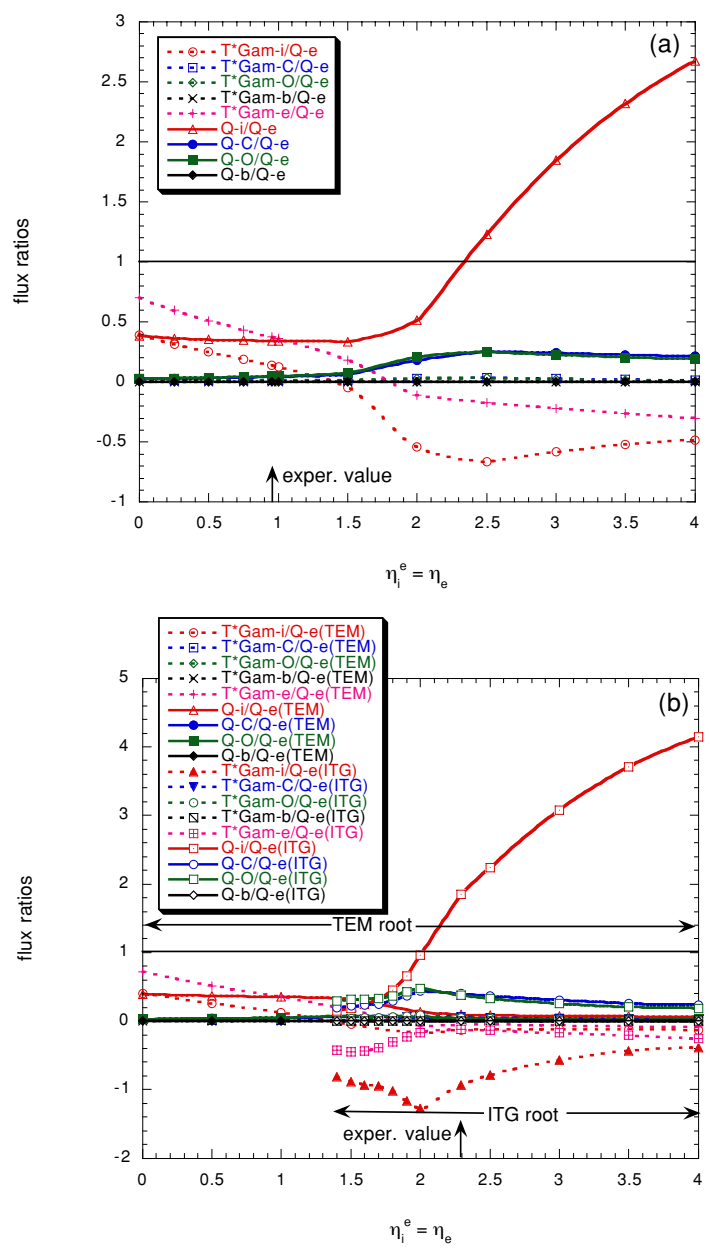

Figure 6. Flux ratios versus $\eta_{i}^{e}=\eta_{e}$ at $s=0.51$ for (a) shot 11849 at $k_{\perp}(\theta=0) \rho_{i}=0.41$ for TEM-ITG root and $(b)$ shot 17120 at $k_{\perp}(\theta=0) \rho_{i}=0.32$. 


\section{External Distribution}

Plasma Research Laboratory, Australian National University, Australia

Professor I.R. J ones, Flinders University, Australia

Professor J oão Canalle, Instituto de Fisica DEQ/IF - UERJ , Brazil

Mr. Gerson O. Ludwig, Instituto Nacional de Pesquisas, Brazil

Dr. P.H. Sakanaka, Instituto Fisica, Brazil

The Librarian, Culham Laboratory, England

Library, R61, Rutherford Appleton Laboratory, England

Mrs. S.A. Hutchinson, JET Library, England

Professor M.N. Bussac, Ecole Polytechnique, France

Librarian, Max-Planck-Institut für Plasmaphysik, Germany

J olan Moldvai, Reports Library, MTA KFKI-ATKI, Hungary

Dr. P. Kaw, Institute for Plasma Research, India

Ms. P.J . Pathak, Librarian, Insitute for Plasma Research, India

Ms. Clelia De Palo, Associazione EURATOM-ENEA, I taly

Dr. G. Grosso, Instituto di Fisica del Plasma, Italy

Librarian, Naka Fusion Research Establishment, J AERI, J apan

Library, Plasma Physics Laboratory, Kyoto University, J apan

Research Information Center, National Institute for Fusion Science, J apan

Dr. O. Mitarai, Kyushu Tokai University, J apan

Library, Academia Sinica, Institute of Plasma Physics, People's Republic of China

Shih-Tung Tsai, Institute of Physics, Chinese Academy of Sciences, People's Republic of China

Dr. S. Mirnov, TRINITI, Troitsk, Russian Federation, Russia

Dr. V.S. Strelkov, Kurchatov Institute, Russian Federation, Russia

Professor Peter Lukac, Katedra Fyziky Plazmy MFF UK, Mlynska dolina F-2, Komenskeho Univerzita, SK-842 15 Bratislava, Slovakia

Dr. G.S. Lee, Korea Basic Science Institute, South Korea

Mr. Dennis Bruggink, Fusion Library, University of Wisconsin, USA

Institute for Plasma Research, University of Maryland, USA

Librarian, Fusion Energy Division, Oak Ridge National Laboratory, USA

Librarian, Institute of Fusion Studies, University of Texas, USA

Librarian, Magnetic Fusion Program, Lawrence Livermore National Laboratory, USA

Library, General Atomics, USA

Plasma Physics Group, Fusion Energy Research Program, University of California at San Diego, USA

Plasma Physics Library, Columbia University, USA

Alkesh Punjabi, Center for Fusion Research and Training, Hampton University, USA

Dr. W.M. Stacey, Fusion Research Center, Georgia Institute of Technology, USA

Dr. J ohn Willis, U.S. Department of Energy, Office of Fusion Energy Sciences, USA

Mr. Paul H. Wright, Indianapolis, Indiana, USA 
The Princeton Plasma Physics Laboratory is operated by Princeton University under contract with the U.S. Department of Energy.

\author{
Information Services \\ Princeton Plasma Physics Laboratory \\ P.O. Box 451 \\ Princeton, NJ 08543
}

Phone: 609-243-2750

Fax: 609-243-2751

e-mail: pppl_info@pppl.gov

Internet Address: http://www.pppl.gov 